\title{
Lightweight Integrated Solar Array (LISA): Providing Higher Power to Small Spacecraft
}

\author{
Les Johnson, ${ }^{1}$ John Carr, ${ }^{2}$ Leo Fabisinski, ${ }^{3}$ and Tiffany Russell Lockett ${ }^{4}$ \\ NASA George C. Marshall Space Flight Center, Huntsville, AL 35801
}

\begin{abstract}
Affordable and convenient access to electrical power is essential for all spacecraft and is a critical design driver for the next generation of smallsats, including CubeSats, which are currently extremely power limited. The Lightweight Integrated Solar Array (LISA), a concept designed, prototyped, and tested at the NASA Marshall Space Flight Center (MSFC) in Huntsville, Alabama provides an affordable, lightweight, scalable, and easily manufactured approach for power generation in space. This flexible technology has many wide-ranging applications from serving small satellites to providing abundant power to large spacecraft in GEO and beyond. By using very thin, ultraflexible solar arrays adhered to an inflatable or deployable structure, a large area (and thus large amount of power) can be folded and packaged into a relatively small volume.
\end{abstract}

\section{Introduction}

$\mathrm{T}$ he CubeSat revolution continues as the number of CubeSats developed and flown by companies, universities and governments continues to rise. As the volume and mass of the spacecraft systems continues to decrease, the demand for power, unfortunately, has not. With the CubeSat form factor, the volume that can be allocated to largesurface area solar arrays is rather small and the cost of using articulated solar arrays, to maximize power output by continuously optimizing the array orientation relative to the Sun, is both expensive and rather risky. There is clearly a need for a low-cost, low-mass, and higher-power alternative to conventional solar arrays for CubeSat and small spacecraft applications.

Thin-film based solar arrays for spacecraft applications have long been recognized as an advantageous power generation option ${ }^{1}$. Thinner materials yield a mass savings, equating to lighter launch loads or more payload allocation. Furthermore, their mechanical flexibility lends itself well to stowage and deployment schemes. Both make thin-film arrays an exciting prospect for small-scale satellites. However, a gap in thin-film array development exists, leaving very few choices for available array structures. The LISA seeks to address this, enabling higher power generation in small spacecraft at low weights and high stowage efficiency without the need for articulation.

The LISA array is a launch-stowed, orbit-deployed structure upon which lightweight photovoltaic devices are embedded. The system will provide a 2.5 to 5.0 fold increase in specific power generation (Watts/kilogram) coupled with a >2x enhancement of stowed volume (Watts/cubic-meter) and a decrease in cost (dollars/Watt) when compared to state-of-the-art solar arrays. Fig. 1 is an artist concept of two LISA units deployed from a smallsat in Low Earth Orbit (LEO). Fig. 2 shows the 2013 laboratory testing of one such module which validated the concept to TRL 4.

\footnotetext{
${ }^{1}$ Technical Assistant, Advanced Concepts Office, NASA MSFC, AL, Mail Code ED04.

${ }^{2}$ Electrical Power Engineer, Space Systems Department, NASA MSFC, AL, Mail Code ES44.

${ }^{3}$ Engineer, Advanced Concepts Office, NASA MSFC, AL, Mail Code ED04.

${ }^{4}$ Mission Analyst, Advanced Concepts Office, NASA MSFC, AL, Mail Code ED04.
} 


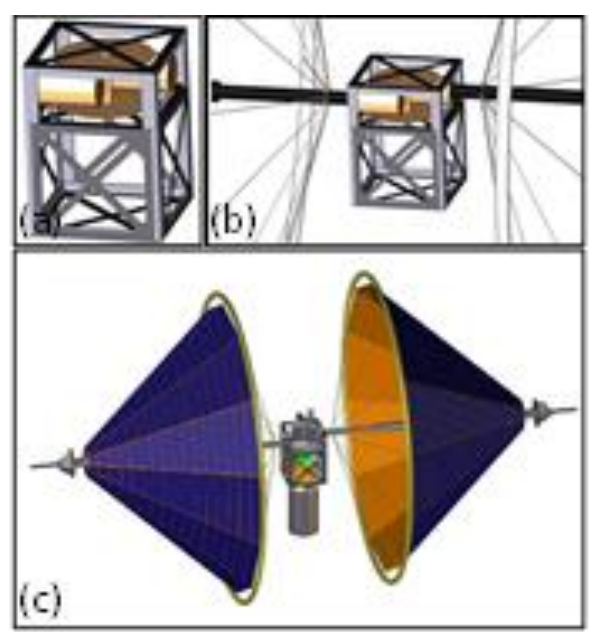

Fig. 1 Conceptual rendering of LISA (a) stowed, (b) and (c) deployed.

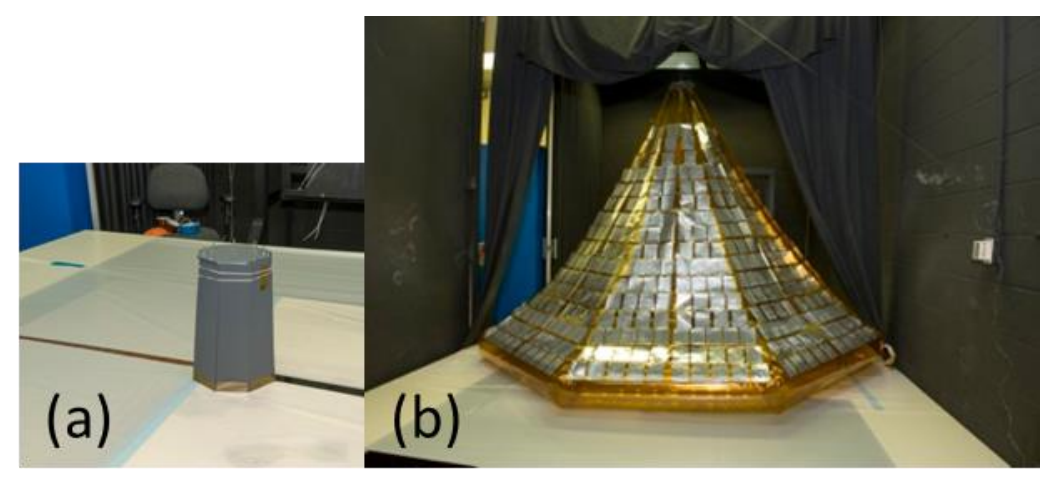

Fig. 2 TRL-4 LISA test article (a) stowed and (b) deployed.

LISA builds upon previously published concepts, such as the PowerSphere ${ }^{2}$, Inflatable Torus Solar Array Technology (ITSAT) ${ }^{3-4}$, and others using new engineering concepts and the latest advancements in thin-film materials, devices, and deployment options. The project leverages several existing and ongoing efforts at Marshall Space Flight Center (MSFC) for design, development, fabrication, and testing. Most notably is extensive solar sail work which is rapidly maturing, lightweight, large area deployment technologies ${ }^{5}$. The most current LISA-T activity will advance the system technology readiness level (TRL) from 4 through $6^{+}$.

\section{The Importance of Geometry and Deployability}

LISA is intended to be deploy and forget. This can only be accomplished if a significant fraction of the total deployed area sees the sun at any orientation, eliminating the need for solar pointing. Different deployment geometries are being considered: cube, cylinder, sphere, triangular pyramid, and torus. Each shape is affected differently by potential packing densities, the configuration knockdown factor (related to projected area and number of deployed sides), as well as the Kelly Cosine Law (Table I). For example, a square side of a cube resembles a more traditional solar panel and cells can be packed to cover $\sim 90 \%$ of the surface area. With an average power generation from a $25 \%$ efficient cell of $\sim 307 \mathrm{~W} / \mathrm{m} 2$ at the module level, $\sim 0.65 \mathrm{~m} 2$ must be illuminated at any given time to produce $200 \mathrm{~W}$ of continuous power. To configure this into a cube, 5 or 6 sides must be deployed to eliminate the need for pointing and, hence, at least $3.25 \mathrm{~m}^{2}$ of array must be stowed and then deployed. Worst-case Kelly Cosine losses are when illumination is normal to one side, as angled incidence will illuminate multiple sides generating more power. The other geometries are summarized in Tab. 1.

Tab. 1 Results from geometric trade study for a deployed array capable of generating $200 \mathrm{~W}$.

\begin{tabular}{|l|l|l|l|l|}
\hline Shape & Packing & Area & Configuration & Total Area \\
\hline
\end{tabular}




\begin{tabular}{|c|c|c|c|c|}
\hline & density $\mathbf{( \% )}$ & $\left(\mathbf{m}^{2}\right)$ & Factor & Required $\left(\mathbf{m}^{\mathbf{2}}\right)$ \\
\hline Cube & 90 & 0.65 & 5.00 & 3.25 \\
\hline Pyramid & 80 & 0.79 & 3.00 & 2.36 \\
\hline Cylinder $^{*}$ & 80 & 0.92 & 3.14 & 3.61 \\
\hline Sphere & 80 & 0.92 & 4.00 & 3.66 \\
\hline Torus $^{* *}$ & 80 & 0.92 & 2.00 & 2.88 \\
\hline
\end{tabular}

In addition to geometry, antenna and photovoltaic integration, ease of fabrication, stowage, and deployment must also be considered. Pathfinder flat-panel ('two-dimensional') articles have been fabricated and tested to help guide initial decision-making (Fig. 3).

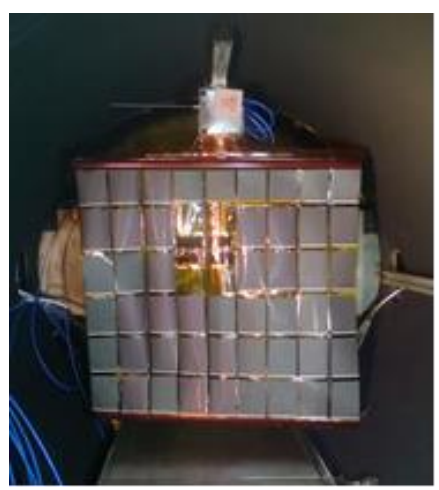

Fig. 3 Pathfinder flat-panel LISA Array. 1U CubeSat with antennas shown at top.

The test article's panels were fabricated from $25 \mu \mathrm{m}$ Kapton with a custom inflation-assist, roll-tube rigidized deployment method. The $\sim 0.45 \mathrm{~m}^{2}$ array included 4 strings of electrically active, un-covered solar cells (remaining cells electrically inactive), electrical routing, and an Ultra High Frequency (UHF) dipole antenna. The array was packaged into $\sim 1 / 3$ of the $1 \mathrm{U}$ skeleton shown at the top and remotely deployed via a burn-wire release mechanism.

Additional deployment options are being considered, including mechanical 'boom-type', inflatable, and hybrid options. Considered in the trade study are stiffness, mass, TRL, reliability, stowed volume, and scalability.

\section{Photovoltaics and Materials}

The field of thin-film photovoltaics is somewhat broad and encompasses technologies from the more traditional, such as amorphous silicon (a:Si) or copper indium gallium (di)selenide (CIGS), to the emerging, such as organic and perovskite based cells. Several technologies are being utilized and traded by the LISA team - each with their own distinct advantages. A metric-based matrix is being built to offer options for different types of missions. For example, a high performance, high reliability mission (superior $\mathrm{W} / \mathrm{m}^{2}, \mathrm{~W} / \mathrm{m}^{3}, \mathrm{~W} / \mathrm{kg}$, and lifetime) with a higher budget (\$/W allocation) can be envisioned. Space-qualified, 30\%+ inverted metamorphic (IMMs) are best suited for such an application. In contrast, a lower budget mission with less emphasis on performance metrics and more emphasis on cost might also be envisioned. Adapted terrestrial market, low cost $(<\$ 20 / \mathrm{W})$ CIGS may be best suited. Both technologies as well as two silicon-based cells are being carried through the current development of LISA. Of course, each option will still enable higher total power generation than current state-of-the-art options.

A similar matrix of options is also being compiled for the supporting and cover materials. For example, a Low Earth Obit (LEO) mission with exposure to atomic oxygen is easily envisioned. Colorless Organic Inorganic Nanocomposite (CORIN), a fluorinated polyimide which NASA has demonstrated to have the highest atomic oxygen resistance of all polymers flown on Materials on the International Space Station Experiment 7 (MISSE 7) ${ }^{6}$, is a well suited substrate for such a case. For higher altitude or deeper space missions, Colorless Polyimide 1 (CP1), a fluorinated, durable polyimide with years of space heritage is being evaluated. Each polyimide can be formulated or coated with vapor deposited materials to exhibit a specific emissivity and alpha, enabling a degree of tunability into the thermal design. 
Cover materials are also included in this matrix. Current trades indicate the short missions LISA is initially being designed for may tolerate flying bare cells. However, it remains clear that longer-term missions would benefit from a protective cover.

Integrating these assembly materials and electrically interconnecting the PV cells is the focus of current efforts. Initial pathfinder samples are being produced on $25 \mu \mathrm{m}$ Kapton via a low outgassing pressure-sensitive adhesive bonding method. Electrical interconnection is currently being accomplished with $12 \mu \mathrm{m}$ copper ribbon bonded via a space rated conductive epoxy. Initial results are promising with less than $2 \%$ (relative) PV degradation through the entire integration process and $0 \%$ loss after repetitive (up to 10x) fold/unfold cycles. The $2 \%$ fabrication loss was exhibited in current only and has been tracked to a light film left atop the cells after the bonding process.

In addition to adhesiveless and polyimide materials, more traditional bonding adhesives are being explored such as the silicon adhesive systems currently utilized by NeXolve in the construction of the James Webb Space Telescope (JWST) sunshade. The team has a significant heritage in space qualifying materials having successfully flight qualified all JWST Sunshield constructions which include adhesiveless seams and bonded assemblies.

Integrated power buses and thin-film wire harnesses are also being developed as a part of the LISA PV assembly. NeXolve's polyimide resins can be applied directly to metallic foils, which can then be patterned and etched to essentially form a multilayer printed circuit board on the assembly substrate; a potential method for interconnecting cells as well as strings to a power bus. A similar concept is also being used to create encapsulated traces on or near the deployment booms - in essence, a thin-film wire harnesses to carry the generated power into the satellite. This significantly reduces weight and enables higher packing efficiency.

\section{Testing}

Subcoupons of the photovoltaic, antenna, and deployment systems are in development and will begin environmental testing this fall. A life cycle test bed will allow integrated power systems to be tested in a simulated space environment (vacuum and thermal) with real-time simulated Air Mass zero (AM0) illumination and power load profiles.

LISA will use the High Intensity Solar Environment Test (HISET) system, which combines the elements of the solar wind environment with a high intensity, high power solar radiation source. The simulated solar wind includes low energy electrons (1-100keV), protons (1-30keV), NUV radiation, and vacuum ultraviolet (VUV) radiation. The pathfinder flat-panel (Fig. 2) was successfully deployed and illuminated in this chamber.

\section{Conclusion}

The LISA project is an innovative approach to generating more power and enabling more communication ability for CubeSats and other small spacecraft. The technology will enable small satellites to perform their missions without having to be power starved or bankrupted. Extensive work is currently underway designing the geometric shape, the deployment mechanisms, stowage, testing the state-of-the-art thin-film PVs, and substrate development. LISA will receive a battery of space environments tests on the component level as well as at the integrated system level. At the end of the current effort a fully tested, TRL 6 qualified engineering unit will be developed.

\section{Acknowledgments}

The authors would like to thank the members of the LISA team for their most excellent technical support on the project: Armando Martinez, Darren Boyd, Michael SanCoucie, Brandon Farmer, Todd Schneider, and Greg Laue. This work would not have been possible without the financing and management support of the NASA MSFC Technology and Investment Program that sponsored the first phase of the research.

\section{References}

\footnotetext{
${ }^{1}$ Bailey, S., et al, "The Future of Space Photovoltaics," in Photovoltaic Specialists Conference (PVSC), 2009, p. 1909.

${ }^{2}$ Simburger, E., et al, "Thin Film Technology Development for the PowerSphere," Materials Science and Engineering: B, 2005, Vol. 116, Iss. 3, p. 265-272.

${ }^{3}$ Malone, P. and Williams, G., "Lightweight Inflatable Solar Array," in Journal of Propulsion and Power, 1996, Vol. 12, No. 5, pp. 866-872.
} 
${ }^{4}$ Malone, P., et al, "Developing A Solar Array," in Small Satellite Conference Technical Session VIII: Subsystems II, 1993.

${ }^{5}$ Alhorn, Dean, et al, "Nano-Sail D: The Small Satellite That Could!," in $25^{\text {th }}$ Annual AIAA/USU Conference on Small Satellites, Technical Session VI, 2011.

${ }^{6}$ Yi, G.T., et al, "Overview of the MISSE 7 Polymers and Zenith Polymers Experiments After 1.5 Years of Space Exposure," in NASA/TM 2013-217848, 2013. 